\title{
Post-operative Radiotherapy Did Not Improve the Treatment Outcomes of Intracranial Hemangiopericytoma
}

\section{Jianbiao Xiao}

Southern Medical University Nanfang Hospital

\section{Yi Ding}

Southern Medical University Nanfang Hospital Department of Spinal Surgery

\section{Lanwei Xu}

Shangdong Provincial Hospital

\section{Wei Wang}

General Hospital of southern theater command

\section{Fen Chen}

Chenzhou No.1 People's Hospital

\section{Xuehui Wu}

Southern Medical University Nanfang Hospital

\section{Yangshu Zhou}

Zhujiang Hospital

\section{Qiyuan Zhou}

Chenzhou No.1 Hospital

\section{Fengjiao Zhang}

Shanghai Concord medical cancer center

\section{Junpeng Li}

general hospital of southern theater command

\section{Li Liang}

Southern Medical University Nanfang Hospital

Yee-Min Jen ( $\nabla$ yeeminjen@gmail.com )

Tri-Service General Hospital https://orcid.org/0000-0001-5869-9940

\section{Research article}

Keywords: intracranial hemangiopericytoma, post-operative radiotherapy, stereotactic radiosurgery, intensity- modulated radiotherapy

Posted Date: June 22nd, 2020 
DOI: https://doi.org/10.21203/rs.3.rs-35658/v1

License: (c) (1) This work is licensed under a Creative Commons Attribution 4.0 International License. Read Full License

Version of Record: A version of this preprint was published at BMC Cancer on August 12th, 2021. See the published version at https://doi.org/10.1186/s12885-021-08594-x. 


\section{Abstract \\ Background}

The purpose of this study is to analyze the impact of post-operative radiotherapy on the results in patients with intracranial hemangiopericytoma (HPC).

\section{Materials and methods}

We retrospectively reviewed 66 intracranial HPC patients between 1999 and 2019 including 29 with surgery followed by radiotherapy (11 with intensity-modulated radiotherapy (IMRT) and 18 with stereotactic radiosurgery (SRS)) and 37 with surgery alone. Chi-squared test was used to compare the clinical characteristic between the groups. The Kaplan-Meier method was used to analyze overall survival (OS) and recurrence-free survival (RFS). Multivariate Cox proportional hazards models were used to examine prognostic factors of survival.

\section{Results}

The crude local control rates were $58.6 \%$ in the surgery plus post-operative radiotherapy group (PORT) and $67.6 \%$ in the surgery alone group $(p=0.453)$. In the subgroup analysis of the PORT patients, local controls were $72.7 \%$ in the IMRT group and $50 \%$ in the SRS group $(p=0.228)$. The median OS in the PORT and surgery groups were 122 months and 98 months, respectively $(p=0.169)$. The median RFS was 96 months in the PORT group and 72 months in the surgery alone group $(p=0.714)$. The median OS and RFS of the SRS group were not significantly better than those in the IMRT group $(p=0.256,0.960)$. The median RFS were 112 and 72 months for pathology grade II and III patients, respectively $(p=0.001)$.

\section{Conclusion}

PORT did not improve the local control rates nor the survivals. The local control rates after IMRT and SRS were similar even though the IMRT technique had a much higher biological dose compared with the SRS technique. PORT is not indicated for intracranial HPC patients with a complete resection margin.

\section{Introduction}

In 1942, Stout AP and Margaret RM described a new type of vascular tumor showing the characteristic formation of endothelial tubes and sprouts surrounded by a sheath of rounded and sometimes elongated cells. They believed that these cells were derived from the capillary pericytes and suggested that the tumors be called hemangiopericytoma (HPC) (1). From then on until 1954, a total of thirty eight cases were reported. In 1954, Begg and Garret reported the first patient of primary cranial meningeal HPC. The tumor histologically resembled both the soft tissue HPC previously described by Stout and Murray and 
the aggressive variant of angioblastic meningioma reported by Cushing and Eisenhardt (2-3). Compared to extracranial HPC, intracranial HPC is less frequent and remains a rare entity representing $0.4 \%$ of all primary central nervous system tumors (4); meningioma is approximately 50 to 60 times more common than intracranial HPC (5-7). It was reported that $1.6-2.5 \%$ of tumors presumed to be meningiomas by neuroimaging were intracranial HPC (8-9). The histological origin of central nervous system HPC has been controversial for a long time, and it is now widely accepted that this tumor arises from the meningeal capillary pericytes. The current WHO classification includes HPC in the group of meningeal mesenchymal non-meningothelial tumors with uncertain malignant potential or borderline malignancy $(10-12)$.

In a study of $191 \mathrm{HPC}$ patients, the authors reported that at first local recurrence, patients who underwent repeated surgery survived longer than those patients who did not (median survival time, 53.0 months vs. 35.7 months; $P=0.028)(13)$. In another report, postoperative radiotherapy (PORT) was shown to reduce local recurrence from $88 \%$ with surgery alone to $12.5 \%$ with PORT (14). Kim et al. demonstrated that routine PORT with 50 to $60 \mathrm{~Gy}$ regardless of the resection margin and histology significantly improved the median time to local recurrence from 19.5 months in those without PORT to 80.5 months in patients with PORT $(p=0.0003)(15)$. Some studies have suggested that complete surgical removal followed by PORT to the tumor bed is the best treatment policy for intracranial HPCs $(8,16-17)$. Others concluded that postoperative external beam radiotherapy (EBRT) to the tumor bed appears to delay recurrence $(8,16)$.

Although adjuvant radiotherapy after surgery has been frequently used in the treatment of HPC, there are no reports comparing conventionally fractionated EBRT and stereotactic radiosurgery (SRS) in HPC. The purpose of this study is to analyze the impact of different PORT techniques (conventionally fractionated EBRT versus SRS) on 66 patients with intracranial HPC.

\section{Materials And Methods}

\section{Patients}

This is a retrospective, observational study. The study protocol was approved by the hospital review board of the five participant hospitals. The medical charts and electronic databases of the hospitals were searched to identify patients diagnosed with primary intracranial HPC who had surgery as the first therapy between 1999 and 2019. Three patients who underwent initial surgery at other hospitals and were later treated for recurrent diseases at one of the five hospitals were also included. All of the patients had pathological confirmation of HPC. Information about their demographic data, pathology, surgery, PORT dose and technique, tumor control, survival status, and treatment-related side effects, were recorded. The pathology slides, when available, were reviewed by one of the authors to confirm the diagnosis.

One of the five participant hospitals used SRS routinely for their intracranial HPC patients. Patients from the other five hospitals were treated with conventionally fractionated intensity-modulated radiotherapy (IMRT) using linear accelerators. The patients were divided into the surgery plus PORT group and the 
surgery alone group; patients in the surgery plus PORT group were further separated into the IMRT group and the SRS group for subgroup analysis. All of the patients were evaluated clinically and radiologically with magnetic resonance imaging (MRI) scans with and without contrast. Follow-up MRI images were compared to the preoperative images by one of the authors and the tumor dimensions, if present, were measured in the axial, sagittal, and coronal planes.

\section{Statistical methods}

The primary endpoint is local tumor control, and secondary endpoints include overall and recurrence-free survivals. All of the statistical analyses were performed by using SPSS v.19. (SPSS Inc., Chicago, IL, USA). Mann-Whitney U-tests (two-tailed) were used to analyze the differences in continuous variables. Fisher's exact test (two-tailed) was used to analyze the differences in categorical variables. Chi-squared test was conducted to compare the differences of clinical characteristics between groups. Kaplan-Meier method was used to analyze local control, overall survival (OS) and recurrence-free survival (RFS). The outcomes were compared between those who receive and did not receive PORT and also between the IMRT and the SRS groups. Univariate and multivariate Cox proportional hazards models were used to search for potential prognostic variables including age ( $\geq 50$ years), gender, tumor location, tumor resection, pathology grade and radiation therapy.

\section{Results}

\section{Patients and Radiotherapy Techniques}

A total of 66 patients diagnosed with intracranial HPC were collected (Table 1). There were 35 males and 31 females with $83.3 \%$ having supratentorial lesions. Gross tumor resection (GTR) was conducted in 61 $(92.4 \%)$ and subtotal tumor resection (STR) in $5(7.6 \%)$ patients. Grade II HPCs were diagnosed in 35 (53\%) patients, and grade III HPCs (anaplastic HPC) were diagnosed in $31(47 \%)$ patients. Twenty-nine $(43.9 \%)$ patients had surgery followed by radiotherapy while thirty-seven $(56.1 \%)$ had surgery alone. The differences in patient characteristics between the two groups with and without PORT were not statistically significant (Table 1). The median follow-up time after operation in all patients was 50.5 months (range, 2-153); it was 57 and 47 months in the surgery plus PORT and the surgery alone groups, respectively. 
Table 1

Characteristics of 66 patients with intracranial hemangiopericytoma by the treatment types.

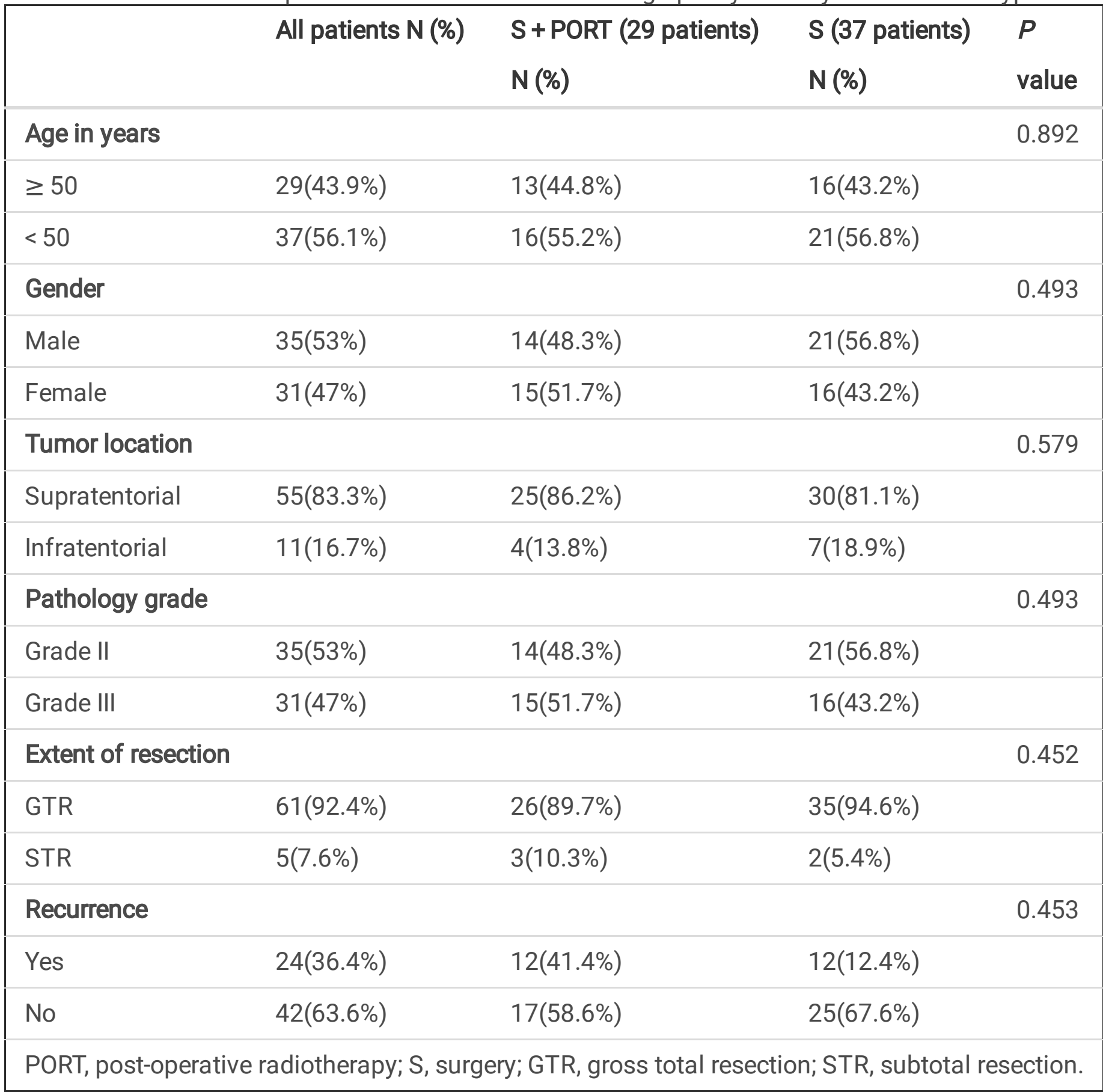

Of the twenty-nine patients with PORT after operation, 11 received IMRT and 18 had SRS (12 had gamma knife SRS and 6 had linac-based SRS) (Table 2). The clinical characteristics were not significantly different between the surgery and PORT groups; they were also similar between the PORT-IMRT and the PORT-SRS groups including the extent of tumor resection and the tumor grade. Eleven patients received fractionated IMRT with a median fraction number of 30 and a median prescription dose of 60 Gy (range 50-60 Gy). IMRT was delivered with 6 MV photons from linear accelerators (Varian Trilogy and Clinac IX; Elekta Synergy). Clinical target volume (CTV) was defined as the tumor cavity and/or the residual mass plus a 5-10 $\mathrm{mm}$ margin. An additional 3-5 $\mathrm{mm}$ was added to the CTV for planning target volume. Eighteen patients underwent gamma knife SRS with a single dose of 14-16 Gy at the margin of tumor 
(12 had gamma knife SRS and 6 had X ray SRS) (Gamma Knife, Elekta, Perfection; Varian Clinac 23ES). At the time of the study cutoff day, 24 of the 29 patients in the PORT group and 25 of 37 patients in the surgery alone group were alive.

Table 2

Characteristics of 29 patients with intracranial hemangiopericytoma receiving post-operative radiotherapy divided by radiotherapy technique

\begin{tabular}{|c|c|c|c|c|}
\hline & All patients $\mathrm{N}(\%)$ & $\begin{array}{l}\text { IMRT (11 patients) } \\
\mathrm{N}(\%)\end{array}$ & $\begin{array}{l}\text { SRS (18 patients) } \\
N(\%)\end{array}$ & $\begin{array}{l}P \\
\text { value }\end{array}$ \\
\hline Age in years & & & & 0.074 \\
\hline$\geq 50$ & $13(44.8 \%)$ & $7(63.6 \%)$ & $6(33.3 \%)$ & \\
\hline$<50$ & $16(55.2 \%)$ & $4(36.4 \%)$ & $12(66.7 \%)$ & \\
\hline Gender & & & & 0.316 \\
\hline Male & $14(48.3 \%)$ & $4(36.4 \%)$ & $8(55.6 \%)$ & \\
\hline Female & $15(51.7 \%)$ & $7(63.6 \%)$ & $8(44.4 \%)$ & \\
\hline Tumor location & & & & 1.000 \\
\hline Supratentorial & $25(84.2 \%)$ & $10(90.9 \%)$ & $15(83.3 \%)$ & \\
\hline Infratentorial & $4(15.8 \%)$ & $1(9.1 \%)$ & $3(16.7 \%)$ & \\
\hline Pathology grade & & & & 0.316 \\
\hline Grade II & $14(48.3 \%)$ & $4(36.4 \%)$ & $10(55.6 \%)$ & \\
\hline Grade III & $15(51.7 \%)$ & $7(63.6 \%)$ & $8(44.4 \%)$ & \\
\hline Extent of resection & & & & 0.862 \\
\hline GTR & $26(89.7 \%)$ & $10(90.9 \%)$ & $16(88.9 \%)$ & \\
\hline STR & $3(10.3 \%)$ & $1(9.1 \%)$ & $2(11.1 \%)$ & \\
\hline Recurrence & & & & 0.228 \\
\hline Yes & $12(41.4 \%)$ & $3(27.4 \%)$ & $9(50 \%)$ & \\
\hline No & $17(58.6 \%)$ & $8(72.7 \%)$ & $9(50 \%)$ & \\
\hline
\end{tabular}

\section{Histological Findings}

All of the 66 patients' pathological examinations with Hematoxylin \& Eosin (H\&E) staining showed an extensively vascularized and cellular tumor. These tumors showed compact and uniform cells with a 
large number of small vascular cavities and compact reticular fibers. Immunohistochemical (IHC) staining showed a strong positivity for CD34. The percentage of ki67 positivity was lower in grade II HPC compared with grade III tumors; the median percentage of positive staining for Ki67 was $2 \%$ (range $1 \%$ $5 \%$ ) in grade II HPC and $12 \%$ in grade III HPC (range 10\% - 16\%). There were more prominent nuclear fission and cell morphology heterogeneity in the higher grade HPC. IHC was negative for PR, S-100 and EM (Fig. 1-1).

\section{Imaging Findings}

All of the patients had MRI examination before and after operation. After craniotomy, MRI was repeated at 3-6-month intervals in the first 3 years with and without contrast. The pre-contrast MRI showed a hypointense lesion on T1 weighted images $(\mathrm{WI})$ and a heterogeneously hyperintense lesion on T2WI. A flow void signal was present in most tumor images, and cystic tumor necrosis and the dural tail sign were also very common. Contrast-enhanced MRI often showed markedly and heterogeneously enhanced lesions. Figures (1-2) showed one of the HPC patient' MRI images before and after operation.

\section{Local Control and Survival}

The crude local control rates were $58.6 \%$ in the surgery plus PORT group and $67.6 \%$ in the surgery alone group ( $p=0.714$ ) (Fig. 2 C). In the subgroup analysis of the PORT patients, they were $72.7 \%(8 / 11)$ in the IMRT group and 50\% $(9 / 18)$ in the SRS group ( $p=0.960)$ (Fig. 2E).

The median RFS in the pathology grade II and III were 112 and 72 months, respectively $(p=0.001)$. Salvage surgery with or without PORT was conducted for most patients with local recurrence (Fig. 2A). The 5-year RFS rates in the surgery plus PORT group and surgery alone group were $56.4 \%$ and $74.6 \%$, respectively.

The median OS in the surgery plus PORT and surgery alone groups were 122 months and 98 months, respectively $(p=0.169)$ (Fig. 2B). The median OS in the SRS and IMRT groups were 127 months and 73 months $(p=0.256)$ (Fig. 2D). The 5-year OS rates in the PORT and surgery alone groups were $75 \%$ and $90.9 \%$, respectively.

\section{Prognostic Factors of OS and RFS}

Age $\geq 50$ years is the only prognostic factor for OS by both the univariate $(p=0.020)$ and multivariate ( $p$ $=0.029$ ) Cox regression analyses (Table 3 ). The median OS time is 84 months in the older group (age $\geq$ 50 years) and 122 months in the younger group (age $<50$ years) $(p=0.018)$. The median RFS time is 72 months in the older group (age $\geq 50$ years) and 96 months in the younger group (age $<50$ years) $(p=$ 0.100 ). 
Table 3

Univariate and Multivariate Analysis of Factors Associated with Overall Survival in Patients of Intracranial Hemangiopericytoma

\begin{tabular}{|lllll|}
\hline \multicolumn{4}{l}{ Univariate Analysis } & \multicolumn{2}{l|}{ Multivariate Analysis } \\
\hline Variable & OS & & OS & \\
\hline Age & $\mathrm{P} 95 \% \mathrm{Cl}$ & & $\mathrm{p}$ & $\mathbf{9} \%$ \\
\hline Gender & $0.020^{*}$ & $0.116-0.834$ & $0.029^{*}$ & $0.103-0.883$ \\
\hline Location & 0.346 & $0.235-1.661$ & 0.573 & $0.406-5.096$ \\
\hline Resection (GTR) & 0.184 & $0.000-5.044$ & 0.963 & 0.000 \\
\hline Recurrence & 0.338 & $0.000-28.840$ & 0.971 & 0.000 \\
\hline Pathology grade & 0.352 & $0.586-4.485$ & 0.348 & $0.136-2.022$ \\
\hline Radiation (Yes) & 0.178 & $0.721-5.869$ & 0.898 & $0.335-3.478$ \\
\hline $\begin{array}{l}\text { OS, Overall survival; Cl, confidence interval; GTR, gross total resection; * P values are statistically } \\
\text { significant. }\end{array}$ & & & & \\
\hline
\end{tabular}

The median RFS in the pathology grade II and III were 112 and 72 , respectively $(p=0.001)$. Pathology grade is the only prognostic factor for RFS by both the univariate $(p=0.003)$ and multivariate $(p=0.005)$ Cox regression analyses (Table 4).

Table 4

Univariate and Multivariate Analysis of Factors Associated with Recurrence-free Survival in Patients of Intracranial Hemangiopericytoma

\begin{tabular}{|lllll|}
\hline \multicolumn{4}{|l}{ Univariate Analysis } & \multicolumn{2}{l|}{ Multivariate Analysis } \\
\hline & RFS & & RFS & \\
\hline Variable & $\mathrm{P} 95 \% \mathrm{Cl}$ & & $\mathrm{p}$ & $95 \% \mathrm{Cl}$ \\
\hline Age & 0.107 & $0.208-1.166$ & 0.083 & $0.173-1.114$ \\
\hline Gender & 0.231 & $0.248-1.400$ & 0.302 & $0.229-1.580$ \\
\hline Location & 0.787 & $0.293-2.538$ & 0.518 & $0.175-2.409$ \\
\hline Resection (GTR) & 0.103 & $0.832-7.404$ & 0.238 & $0.591-8.314$ \\
\hline Pathology grade & $0.003^{*}$ & $1.639-10.663$ & $0.005^{*}$ & $1.495-9.813$ \\
\hline Radiation (Yes) & 0.716 & $0.375-1.961$ & 0.781 & $0.463-2.783$ \\
\hline RFS, Recurrence free survival; Cl, confidence interval; GTR, gross total resection; * P values $<0.5$ \\
\hline
\end{tabular}




\section{Discussion}

Intracranial HPC is a rare disease, and it is also rare in the literature for any single study having a large case number and a satisfactory length of follow-up. In our present report on 66 patients, some of our findings concur with the previously published results in the literature, but some do not. Impact of the extent of tumor resection have been examined by several papers, and complete tumor resection was shown to play a pivotal role for both local control and survival $(6,13,18-22)$. In our study, however, GTR did not affect the treatment results. This may be explained by that $90 \%$ of our patients had GTR and thus making it difficult to detect a difference statistically when compared with the small number of patients with residual tumor.

Most studies investigating the effect of PORT in intracranial HPC were based on single center analysis with a limited patient number, and the results were often contradictory. Meta-analyses and studies based on accumulated database have been conducted to overcome the problems of small case series, but their results were also inconsistent (23-25). Although the role of PORT in the GTR patients is not clear, the general consensus is that PORT is beneficial for patients undergoing STR. Some studies found that PORT following STR improves both RFS and OS compared with STR alone $(7,24-27)$, and the others reported that PORT following GTR may also prolong OS $(23,25,27,28)$ or improve local control $(29,30)$. Contrary to the above, some authors have reported that PORT after GTR has no impact on survival $(5,24,31)$ or that PORT should not be used except for patients with recurrences $(32,33)$. In our study, we have found that PORT after surgery has no significant impact on the overall and disease-free survivals. This is in line with the results from Lee et al who underwent an analysis of practice pattern in the US for intracranial HPC and the PORT effect on its survival. They obtained data of 588 cases from the cancer registry, of which 323 (54.9\%) received postoperative radiation. The 5-year overall survival for those receiving PORT $77.1 \%$, not significantly different from the $83.8 \%$ for those who did not $(p=0.14)$. Postoperative radiation was not prognostic for survival on multivariable analysis (34).

SRS has been used for the patients with residual or recurrent intracranial HPCs (17, 32, 35-39). It was reported that postoperative SRS resulted in a better local tumor control in intracranial HPC patients (17). In our subgroup analysis, patients with SRS had a similar OS to the patients with IMRT. The biologically effective dose (BED) of the SRS and IMRT groups for HPC with an $\alpha / \beta$ ratio of 10 Gy was about 33.6$41.6 \mathrm{~Gy}_{10}$ and $72 \mathrm{~Gy}_{10}$, respectively. The biological effectiveness of the IMRT technique was much higher than the SRS method, yet resulting in no higher local control. The reason is not clear, but considering that SRS may be able to minimize the radiation to the adjacent tissues due to the high-precision delivery of radiation to HPC with a steep radiation dose gradient, it is a reasonable PORT option for HPC patients (32, 40). There is no consensus on the optimal radiation dose for single-fraction SRS for intracranial HPC. Some centers have reported an improved local control at higher tumor margin doses, ranging from 14 to 17 Gy $(41,42)$, but other studies using tumor margin doses exceeding 20 Gy did not show an improved local control $(43,44)$. 
From our analysis, PORT does not seem to improve local control, RFS and overall survival. It should not be given to intracranial HPC patients without a positive margin. The weakness of this study includes the retrospective nature and a small sample size. To examine the exact impact of PORT on local control and survival and the different effects between IMRT and SRS as a PORT option requires a multi-center randomized trial with a larger sample size.

\section{Data sharing statement:}

All of the data are presented in this article except the MRI and pathological images which are available on request.

\section{Declarations}

\section{Sources of support:}

This work had been supported by National Natural Science Foundation of China (No. 81872041, 81672821, 81872470).

\section{Conflict of Interest statement:}

The authors have no conflicts of interest

\section{Data sharing statement:}

All of the data are presented in this article except the MRI and pathological images which are available on request.

\section{Compliance with Ethical Standards:}

\section{Funding:}

This study was funded by National Natural Science Foundation of China (No. 81872041, 81672821, 81872470).

\section{Conflict of interest:}

All the authors declare no conflict of interest.

\section{Ethical approval:}

The study protocol was approved by the hospital review boards of the participant hospitals with a waiver of informed consent of the individual patients. The study was conducted in accordance with the ethical standards of the institutional and national research committees and with the 1964 Helsinki declaration and its later amendments of comparable ethical standards. 


\section{References}

1. Arthur PS, Margaret RM. Hemangiopericytoma: a vascular tumor featuring zimmermann's pericytes. Ann Surg. 1942;116:26-33.

2. Cushing $\mathrm{H}$, Eisenhardt L. Meningiomas: their classification regional behaviour life history and surgical results. Arch NeurPsych. 1939;41(3):657-8.

3. Begg CF, Garret R. Hemangiopericytoma occurring in the meninges: case report. Cancer. 1954;7(3):602-6.

4. Bonde VR, Goel A. Two patients with intracavernous hemangiopericytoma. J Clin Neurosci. 2009;16:330-3.

5. Louis DN, Ohgaki H, Wiestler OD, et al. The 2007 WHO classification of tumours of the central nervous system. Acta Neuropathol. 2007;114:97-109.

6. Rutkowski MJ, Jian BJ, Bloch 0 , et al. Intracranial hemangiopericytoma: clinical experience and treatment considerations in a modern series of 40 adult patients. Cancer. 2012;118:1628-36.

7. Melone AG, D'Elia A, Santoro F, et al. Intracranial Hemangiopericytoma - Our Experience in 30 Years: A Series of 43 Cases and Review of the Literature. World Neurosurg. 2014;81:556-62.

8. Guthrie BL, Ebersold MJ, Scheithauer BW, et al. Hemangiopericytoma -- the role of radiotherapy, treatment, and long-term follow-up of 44 cases. Neurosurgery. 1989;25:514-22.

9. Jellinger K, Slowik F. Histological subtypes and prognostic problems in meningiomas. J Neurol. 1975;208:279-98.

10. Kleihues P, Burger PC, Scheithauer BW. World Health Organization International Histological Classification of Tu mours: histological typing of tumours of the central nervous system. Berlin Heidelberg New York Tokyo: Springer; 1991. p. 38.

11. Kleihues P, Cavenee WK. WHO Classification of tumors of the nervous system. Pathology and genetics of tumors of the nervous system. Lyon: International Agency for Research of Cancer; 2000. pp. 6-7.

12. Alen JF, Lobato RD, Gomez PA, et al. Intracranial Hemangiopericytoma: Study of 12 Cases. Acta Neurochir. 2001;143:575-86.

13. Wang W, Zhang GJ, Zhang LW, et al. Long-Term Outcome and Prognostic Factors After Repeated Surgeries for. Intracranial Hemangiopericytomas World Neurosurg. 2017;107:495-505.

14. Sheehan J, Kondziolka D, Flickinger J, et al. Radiosurgery for treatment of recurrent intracranial hemangiopericytomas. Neurosurgery.2002;51:905 - 11.

15. Kim YJ, Park JH, Kim Yl, et al. Treatment Strategy of Intracranial Hemangiopericytoma. Brain Tumor Res Treat. 2015;3:68-74.

16. Jellinger K, Paulus W, Slowik F. The enigma of meningeal hemangiopericytoma. Brain Tumor Pathol. 1991;8:33-43. 
17. Kano H, Niranjan A, Kondziolka D. Adjuvant stereotactic radiosurgery after resection of intracranial hemangiopericytomas. Int J Radiation Oncology Biol Phys. 2008;72:1333-9.

18. Dufour $\mathrm{H}$, Métellus $P$, Fuentes $\mathrm{S}$, et al. Meningeal hemangiopericytoma: a retrospective study of 21 patients with special review of postoperative external radiotherapy. Neurosurgery. 2001;48:756-62.

19. Fountas KN, Kapsalaki E, Kassam M, et al. Management of intracranial meningeal hemangiopericytomas: outcome and experience. Neurosurg Rev. 2006;29:145-53.

20. Heiser MA, Waldron JS, Tihan T, et al. Temporal fossa hemangiopericytoma: a case series. Otol Neurotol. 2009;30:985-9.

21. Kim JH, Jung HW, Kim YS, et al. Meningeal hemangiopericytomas: long-term outcome and biological behavior. Surg Neurol. 2003;59:47-53.

22. Kumar N, Kumar R, Kapoor R, et al. Intracranial meningeal hemangiopericytoma: 10 years experiences of a tertiary care institute. Acta Neurochir. 2012;15:1647-51.

23. Sonabend AM, Zacharia BE, Goldstein $\mathrm{H}$, et al. The role for adjuvant radiotherapy in the treatment of hemangiopericytoma: a surveillance, epidemiology, and end results analysis. J Neurosurg. 2014;120:300-8.

24. Ghia AJ, Allen PK, Mahajan A, et al. Intracranial hemangiopericytoma and the role of radiation therapy: a population based analysis. Neurosurgery. 2013;72:203-9.

25. Stessin AM, Sison C, Nieto J, et al. The role of postoperative radiation therapy in the treatment of meningeal hemangiopericytoma-experience from the SEER database. Int J Radiat Oncol Biol Phys. 2013;85:784-90.

26. Staples JJ, Robinson RA, Wen BC, et al. Hemangiopericytoma-the role of radiotherapy. Int J Radiat Oncol Biol Phys. 1990;19:445-51.

27. Schiariti M, Goetz P, El-Maghraby H, et al. Hemangiopericytoma: long-term outcome revisited. J Neurosurg. 2011;114:747-55.

28. Soyuer S, Chang EL, Selek U, et al. Intracranial meningeal hemangiopericytoma: the role of radiotherapy: report of 29 cases and review of the literature. Cancer. 2004;100:1491-7.

29. Kim JH, Jung HW, Kim YS, et al. Meningeal hemangiopericytomas: long term outcome and biological behavior. Surg Neurol. 2003;59:47-53.

30. Ghia AJ, Chang EL, Allen PK, et al. Intracranial hemangiopericytoma: patterns of failure and the role of radiation therapy. Neurosurgery. 2013;73:624-30.

31. Rutkowski MJ, Sughrue ME, Kane AJ, et al. (2010) Predictors of mortality following treatment of intracranial hemangiopericytoma. J Neurosurg. 2010;113:333-339.

32. Olson $\mathrm{C}$, Yen $\mathrm{CP}$, Schlesinger $\mathrm{D}$, et al. Radiosurgery for intracranial hemangiopericytomas: outcomes after initial and repeat Gamma Knife surgery. J Neurosurg. 2010;112:133-9.

33. Rutkowski MJ, Bloch 0 , Jian BJ, et al. Management of recurrent intracranial hemangiopericytoma. J Clin Neurosci. 2011;18:1500-4. 
34. Lee A, Sidiqi B, Wang A, et al. Patterns of care and outcomes of postoperative radiation for intracranial hemangiopericytoma in United States hospitals. Clin Neurol Neurosurg. 2018;167:1-5.

35. Payne BR, Prasad D, Steiner M, et al. Gamma surgery for hemangiopericytomas. Acta Neurochir. 2000;142:527-36.

36. Ecker RD, Marsh WR, Pollock BE, et al. Hemangiopericytoma in the central nervous system: treatment, pathological features, and long-term follow up in 38 patients. J Neurosurg. 2003;98:1182-7.

37. Sun S, Liu A, Wang C. Gamma Knife radiosurgery for recurrent and residual meningeal hemangiopericytomas. Stereotact Funct Neurosurgery. 2009;87:114-9.

38. Or Cl, Lee CC, Seyed HM, et al. Stereotactic radiosurgery for intracranial hemangiopericytomas: a multicenter study. J Neurosurg. 2017;126:744-54.

39. Jin WK, Dong GK, Chung HT, et al. Gamma Knife stereotactic radiosurgery for intracranial hemangiopericytomas. J Neurooncol. 2010;99:115-22.

40. Ganz JC. Rare tumours and other lesions. In: Ganz JC, editor. Gamma knife neurosurgery. Wien: Springer; 2011. pp. 349-52.

41. Kano H, Niranjan A, Kondziolka D, et al. Adjuvant stereotactic radiosurgery after resection of intracranial hemangiopericytomas. Int J Radiat Oncol Biol Phys. 2008;72:1333-9.

42. Kim JW, Kim DG, Chung HT, et al. Gamma knife stereotactic radiosurgery for intracranial hemangiopericytomas. J Neurooncol. 2010;99:115-22.

43. Chang SD, Sakamoto GT. The role of radiosurgery for hemangiopericytomas. Neurosurg Focus. 2005;14(5):14.

44. Veeravagu A, Jiang B, Patil CG, et al. Cyber knife stereotactic radiosurgery for recurrent, metastatic, and residual hemangiopericytomas. J Hematol Oncol. 2011;4:26.

\section{Figures}


H\&E staining

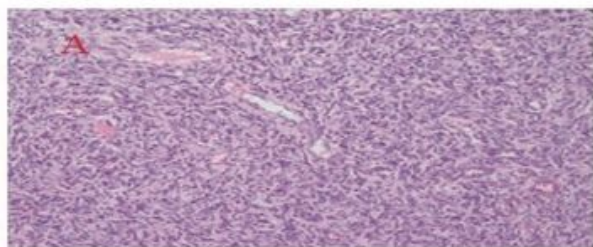

$$
\text { B }
$$

CD 34

Ki 67

1-2
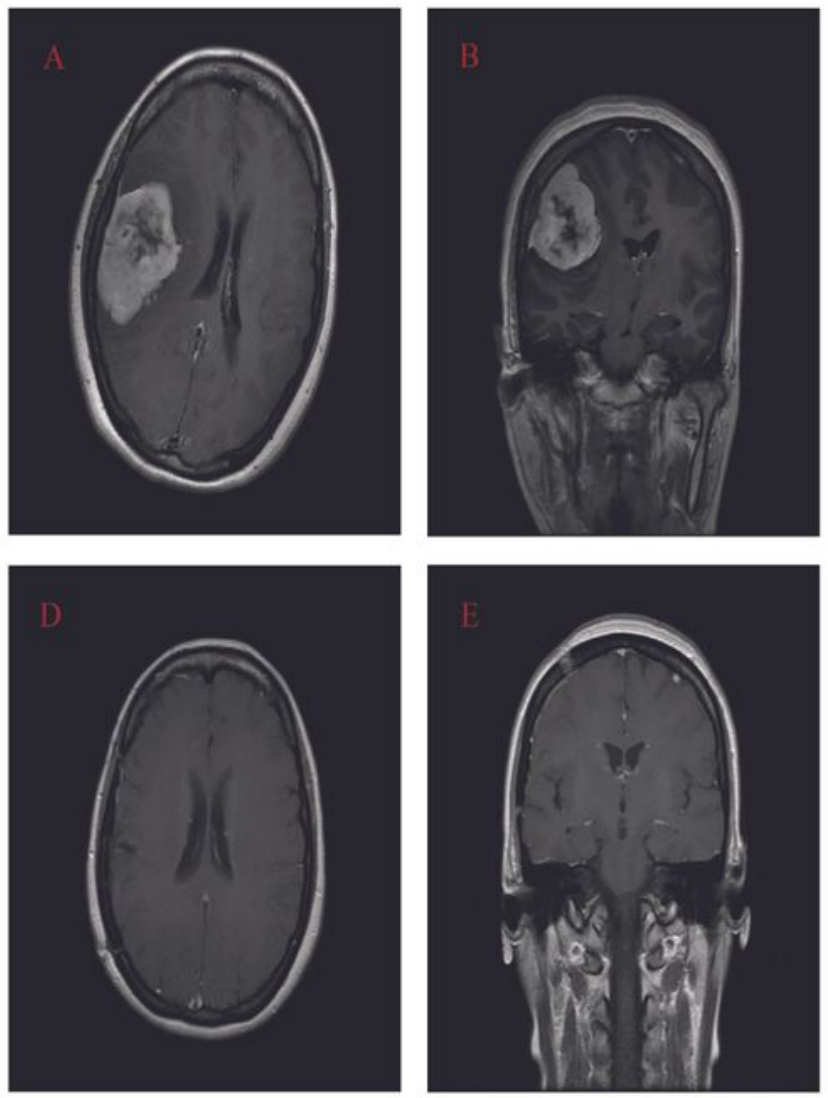

Grade III
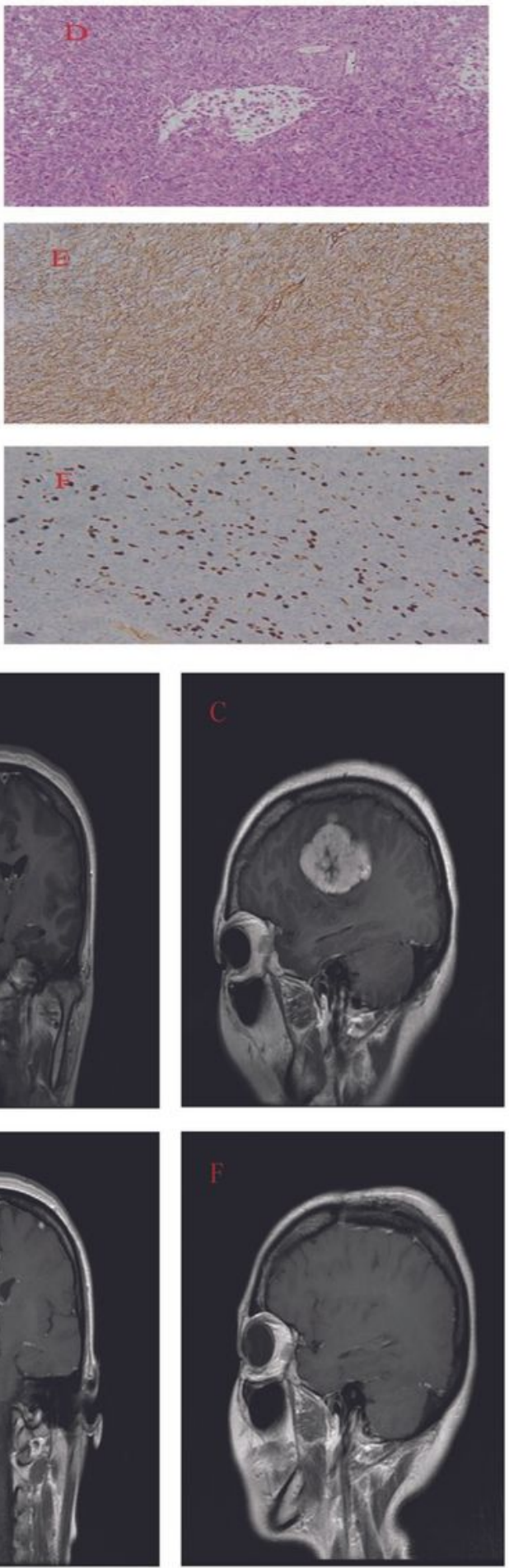

\section{Figure 1}

Figure (1-1). A, B and C: Microphotographs of the pathologic images of a grade II intracranial hemangiopericytoma patient (magnification $x 200$ ). A, Hematoxylin \& Eosin staining demonstrated an extensively vascularized and cellular tumor; $B$, Immunohistochemical staining showed a strong positivity for CD34; C, immunohistochemical staining showed a $5 \%$ positivity for Ki67; D, E and F: Microphotographs of the histologic images of a grade III intracranial hemangiopericytoma patient 
(magnification x200). D, Hematoxylin \& Eosin staining demonstrated an extensively vascularized and cellular tumor; $E$, Immunohistochemical staining showed a strong positivity for CD34; $F$, Immunohistochemical staining showed a 10\% positivity for Ki67. Compared with grade II HPC, the grade III tumor showed a higher percentage of ki67 positivity and more prominent nuclear fission and cell morphology heterogeneity. Figure (1-2). Magnetic resonance imaging (MRI) of a grade II intracranial hemangiopericytoma patient without recurrence after gross total resection. (A-C): Preoperative T1weighted MRI scans with contrast: (A) axial, (B) coronal and (C) sagittal images, showing an enhanced lesion at the right temporal lobe with central necrosis. (D-F) Postoperative T1-weighted MRI scans with contrast: (D) axial, (E), coronal, and (F), sagittal images, showing a complete tumor removal. The patient was recurrence-free twenty-two months after surgery. His histologic images were shown in figure 1-1. 

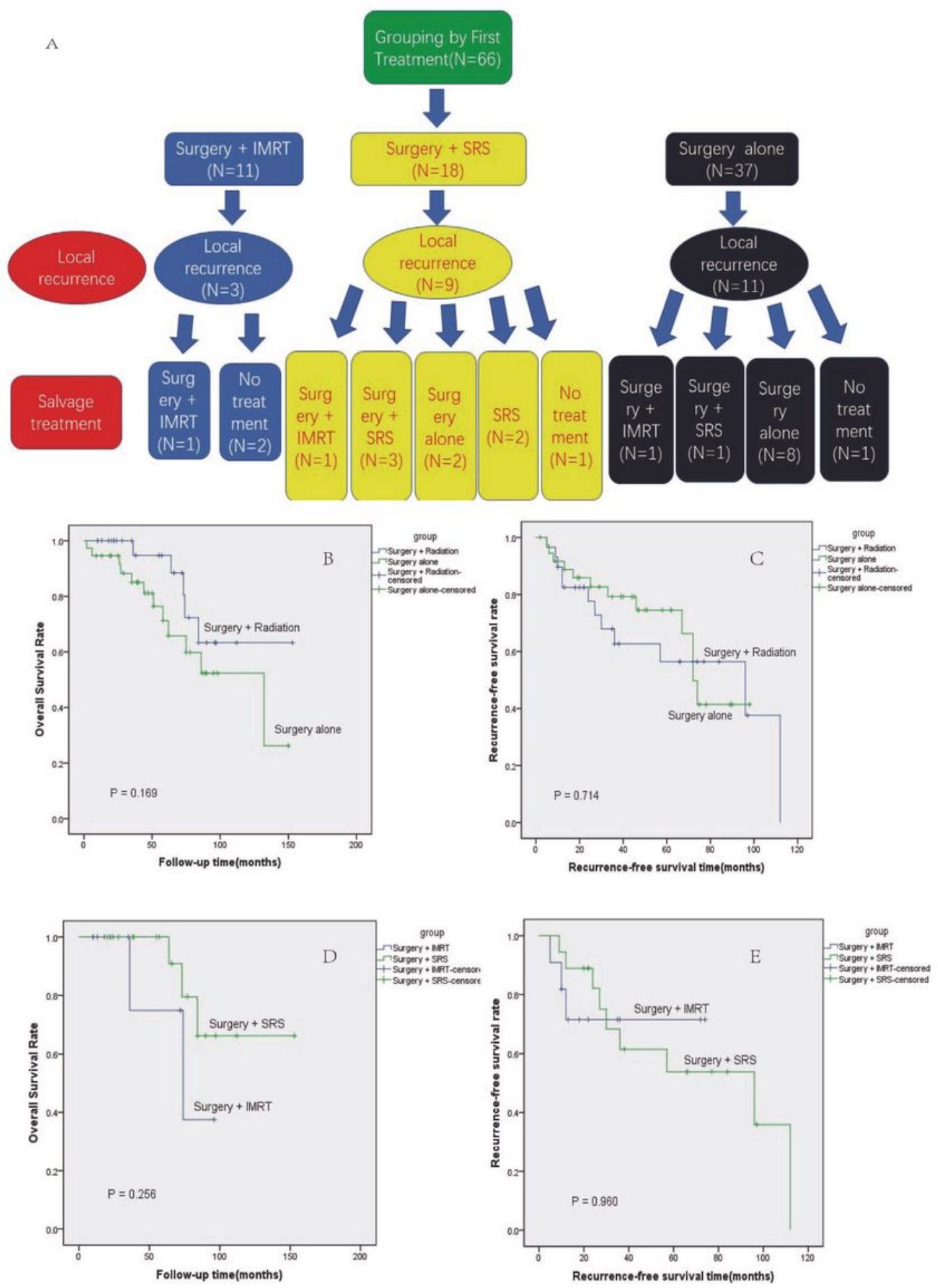

\section{Figure 2}

(A) A diagram showing the initial patient treatments, local recurrence and salvage therapy after local recurrence. Twenty-nine (43.9\%) patients had surgery followed by post-operative radiotherapy (PORT) while thirty-seven (56.1\%) had surgery alone. Of the 29 patients with PORT after operation, 11 received intensity-modulated radiotherapy and 18 had stereotactic radiosurgery (SRS) (12 had gamma knife SRS and 6 had linac-based SRS). There were 12 local recurrences out of the 29 patients with surgery plus 
PORT, and 11 of the 37 surgery alone patients. (B) Kaplan-Meier estimates of the overall survival (OS) curves of intracranial hemangiopericytoma patients. There were no differences between the patients with and without PORT ( $p=0.169$ ). (C) Kaplan-Meier estimates of the recurrence-free survival (RFS) curves in intracranial hemangiopericytoma patients. Postoperative radiotherapy did not increase the RFS

$(p=0.714)$. (D) Kaplan-Meier estimates of the OS curves in intracranial hemangiopericytoma patients with PORT. The OS of the two groups were similar $(p=0.256)$. (E) Kaplan-Meier estimates of the RFS curves in intracranial hemangiopericytoma patients with PORT. The RFS of the two groups were similar $(p=0.960)$.

\section{Supplementary Files}

This is a list of supplementary files associated with this preprint. Click to download.

- coverletterhpcBMCcancer2020610.docx 